\title{
Anesthesia Related Closed Claims and Litigations at the Detroit Medical Center: Analysis, Lessons Learned, and Conclusions
}

\author{
Kris Ferguson, Jason Young, Peter Panagopoulos, Philip Khoury, George M. McKelvey, \\ Samir F. Fuleihan, Walid A. Osta* \\ Department of Anesthesiology, Wayne State University/Detroit Medical Center, Detroit, USA \\ Email: *walidosta@hotmail.com
}

Received 24 January 2014; revised 8 March 2014; accepted 26 March 2014

Copyright (C 2014 by authors and Scientific Research Publishing Inc.

This work is licensed under the Creative Commons Attribution International License (CC BY).

http://creativecommons.org/licenses/by/4.0/

(c) (i) Open Access

\begin{abstract}
The analysis of closed claims and litigations can provide an invaluable tool to improve patient safety by minimizing adverse anesthesia-related outcomes. Analysis of collective data describing such claims is integral to develop new guidelines aimed to reduce adverse anesthesia-related events. In this study, we give a descriptive analysis of anesthesia-related claims at the Detroit Medical Center, Detroit, MI, USA. The study analyzed different components in anesthesia-related closed claims and litigations such as medical, demographic and socio-economic factors. From 67,000 procedures in anesthesiology care provider, related cases claims were made in $0.057 \%$ $(38 / 67,000)$ of all cases. The majority of claims involved procedures involving Caucasian females aged 51 - 55 years. The highest risk periods involved early shift times during Monday and Tuesday, particularly of procedures performed during August. About 33\% of all cases in which death occurred involved patients who received an Obstetrics/Gynecology or an Orthopedic procedure under general anesthesia. The majority of closed claims and litigations cases were distributed between procedures treating nerves injuries and anoxic encephalopathy. The OR and PACU at urban hospitals had the highest claim rates. MD anesthesiologists constituted the lowest proportion of all anesthesia providers involved in closed claims incidents. The average compensation paid was predominately in the range of $\$ 200,000-\$ 250,000$. In addition to reporting anesthesia related factors involved in closed claims and litigations this study also includes a series of recommendations which may work as a framework for improving anesthesia practices.
\end{abstract}

\section{Keywords}

Anesthesia, Closed Claims, Complications, Litigations, Malpractice, Negligence

\footnotetext{
${ }^{*}$ Corresponding author.

How to cite this paper: Ferguson, K., et al. (2014) Anesthesia Related Closed Claims and Litigations at the Detroit Medical Center: Analysis, Lessons Learned, and Conclusions. Open Journal of Anesthesiology, 4, 88-98.

http://dx.doi.org/10.4236/ojanes.2014.44014
} 


\section{Introduction}

The use of regional and general anesthesia and analgesia can result in various adverse complications. These adverse outcomes are often an inherent risk of medical care and do not necessarily reflect negligence [1] [2]. Despite strict adherence to practicing best standard of patient care, anesthesia-related adverse events can result in medico-legal claims.

The term medical liability claim refers to a claim that an action in medical practice deviates from acceptable standards, presumably resulting in the death or injury of a patient, entitling legal responsibly before the law [1]. These incidents are usually tracked and evaluated in many countries using set standards [3]-[6]. The American Society of Anesthesiologists Closed Claims Project (ASA-CCP) reveals data obtained from the American medical liability insurance companies regarding outcomes of anesthesia-related closed claims [7]. ASA-CCP data is gathered collectively without emphasis on a specific institution or group of practitioners. Each year, one or more anesthesiologists are named as defendants in anesthesia related litigations at the Detroit Medical Center (DMC). These litigations may end up dismissed, settled, or trialed. Often, such litigations result in financial restitution to the patient and often can lead to changes in policies and improvement of medical procedures at our institution.

It is of interest to conduct an analysis regarding closed claims and litigations at our medical facilities in order to understand the patterns of the litigation and the distribution of factors involving malpractice cases in order that improvement in anesthetic care, where required, is implemented.

\section{Methods}

Study Design: A retrospective chart review designed to review and collect data from electronic and paper charts from the DMC. As data was also collected from insurance companies in regards to claims and litigation using non-private information that is available to the public institutional IRB approval was not required in this study.

Inclusion/Exclusion Criteria: Inclusion Criteria included litigation cases that involved an anesthesiologist at DMC during the period from January 2004 until December 2009. Exclusion criteria included any litigation cases of medical records/records with incomplete information.

For Each Claim: The case-related medical records, the outcome reports, the testimonies from involved medical providers, witnesses, and experts, the summary of depositions, as well as the settlements cost or jury award were reviewed. Information regarding patient demographics (age, gender, and race), medical charts (height, weight, past medical and surgical history, current medications, and allergies), surgical procedure, anesthetic management, sequence of events and their locations, critical incidents and manifestations of injury, and patient's outcome (morbidity/mortality) were recorded.

\section{Statistics}

As this study was predominately descriptive, results were presented as either numbers of individual events or a percentage of total events.

\section{Results}

During the period between 2004 and 2009, 38 anesthesia-related closed claims and litigation cases were reported. The total number of cases involving hospital performed anesthesia cases was approximately 67,000 cases during the study time duration (2004-2009) with the number of cases performed per year remaining relatively constant. The overall rate of malpractice cases was $0.0567 \%$ during this period. The number of cases for each year $2004-$ 2009 were 7, 10, 6, 5, 8 and 1 respectively. The peak number of cases occurred in 2005 and then decreased to their lowest level of one case in 2009.

\subsection{Patient Demographics}

The majority of cases took place in the suburb hospitals (Figure 1). Of the total of 38 litigation cases were filed, 11 (29\%) involved male patients and 27 (71\%) involved female patients. The age range of the patients involved in litigation was between 23 and 78. Six of the 38 claimants ages was not disclosed. Approximately 25\% ( $\mathrm{n}=8$ ) of claims occurred within the 20 - $40 \mathrm{yr}$ age demographic; 50\% (n=16) occurring within the 40 - $60 \mathrm{yr}$ demographic and 25\% $\mathrm{n}=7$ occurring in the $60-85 \mathrm{yr}$ demographic. The highest proportion of claims occurred in patients between 51 and 55 years of age; 25\% $(n=8)$ (Figure 2). 


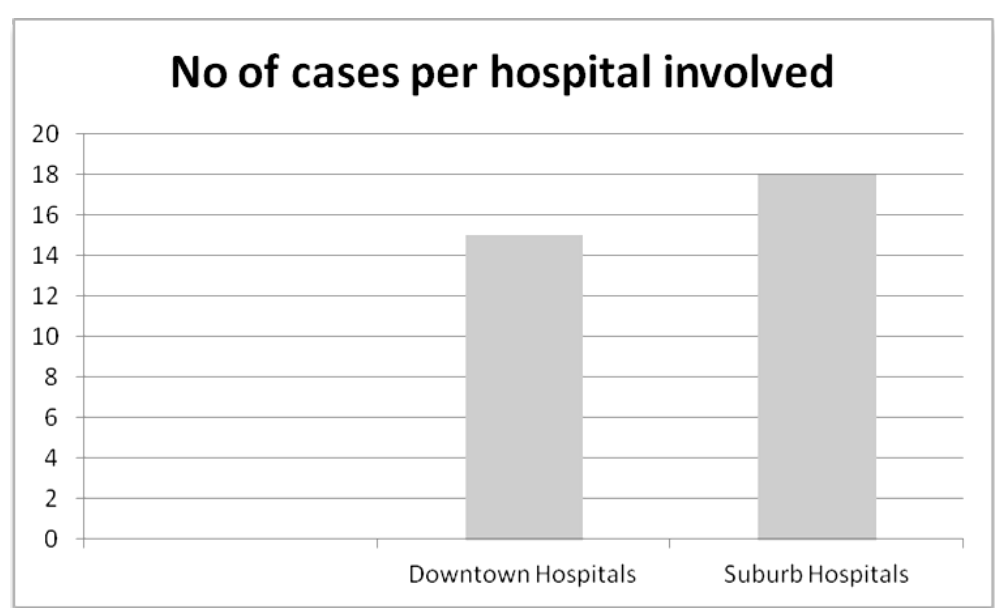

Figure 1. Litigation claims involving anesthesia care provider distribution among Hospital location affiliated with DMC.

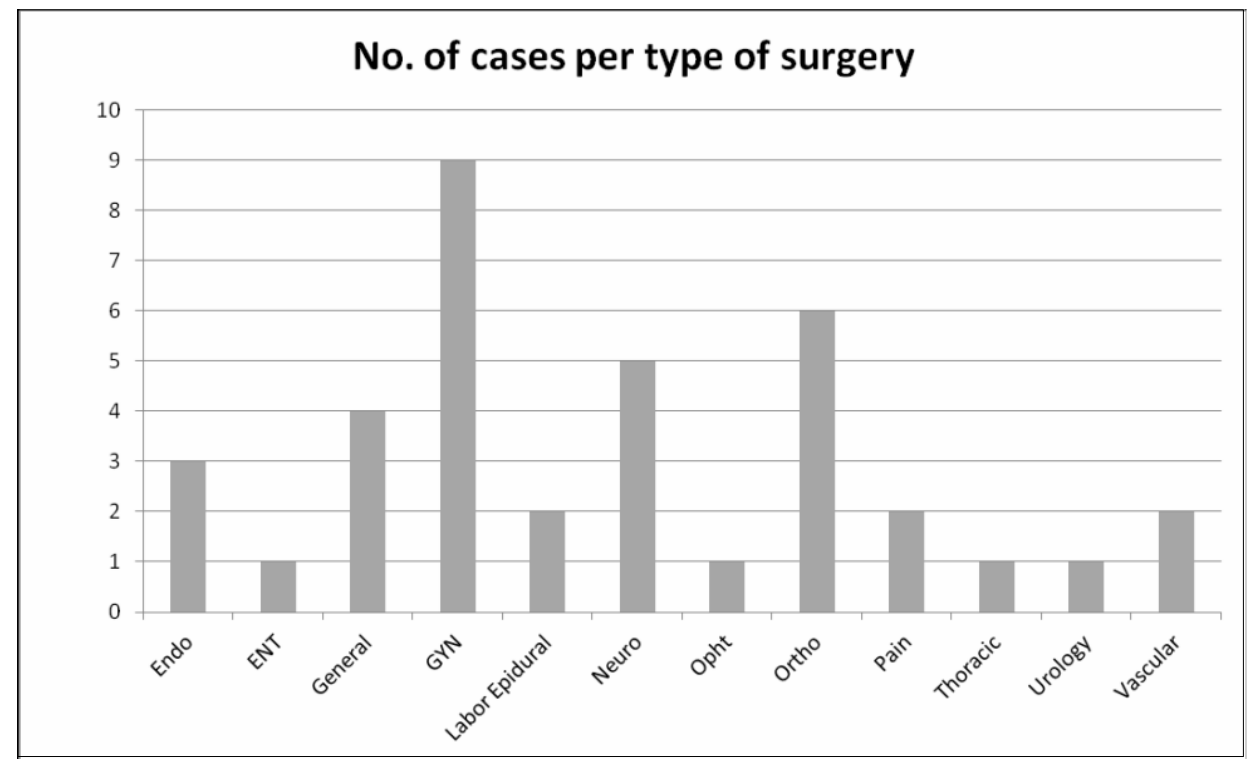

Figure 2. Litigation claims involving anesthesia care provider distribution among surgery types at DMC. Endo-Endoscopy, ENT-Otolaryngology related surgery, GYN-Obstetrics and Gynecology, Neuro-Neurosurgery, Opht-Ophthalmology surgery, Ortho-Orthopedic surgery, Pain-Interventional pain procedure.

The majority of people who reported their race as White/Caucasian were of middle class socio-economic status.

\subsection{Location and Time}

The majority of litigation related injuries occurred in the operating room and PACU (66\%; Figure 3). The remainder took place on the floor (17\%), at home (14\%), and in the ICU (3\%). With the exception of 3 incidents that occurred on the weekend, the majority of incidents (92\%) were reported during the regular business week (Monday-Friday) with Mondays $(\mathrm{n}=7 ; 18 \%)$ and Tuesdays $(\mathrm{n}=11 ; 29 \%)$ having the highest proportion of incidents. With the exception of 1 case, all incidents were reported to occur during the standard business hours of operation between 06:00 and 15:00 with the most common time of the injury between 06:00 and 09:00. For the time of year in which incidents occurred approximately one-third of the 38 incidents reported occurred during the months of August, and September, with August n = 7; 18\%) having the highest incidence. 


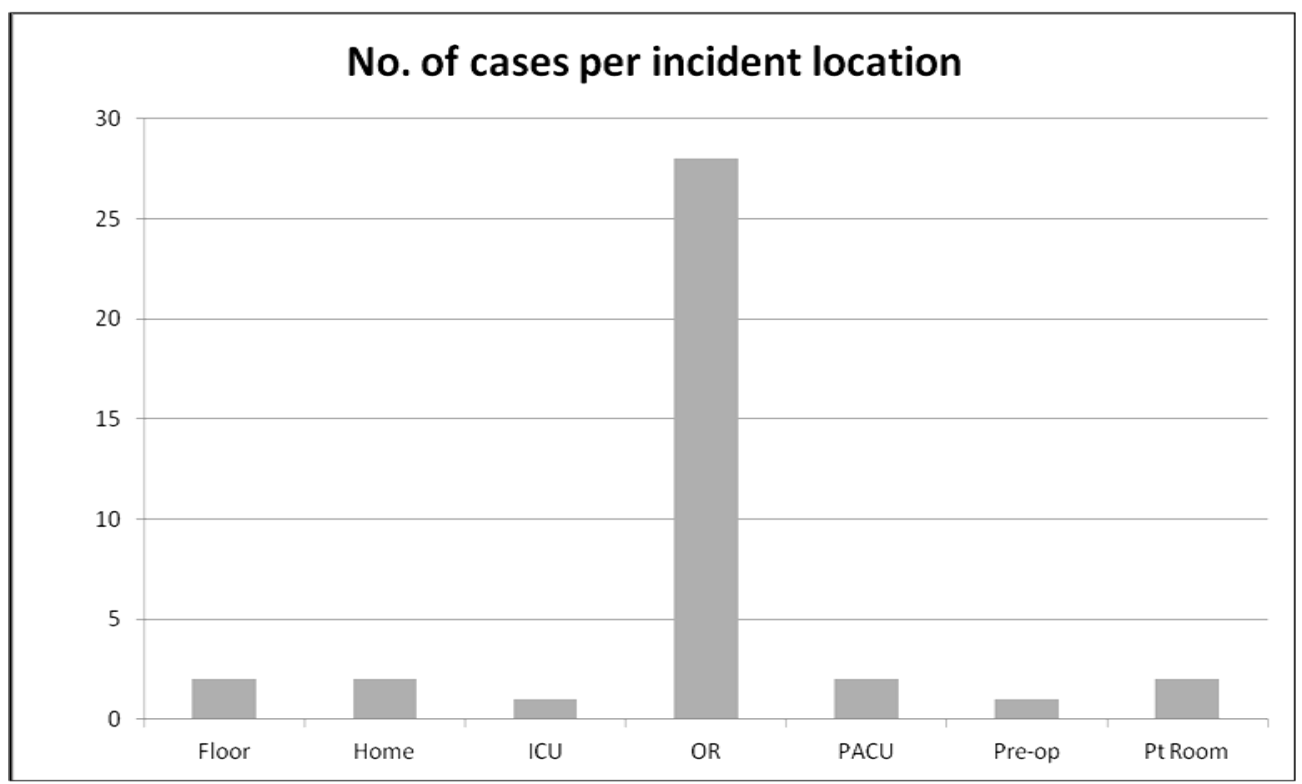

Figure 3. Litigation claims involving anesthesia care provider distribution among care providing locations at DMC. Add a key giving the full title for any abbreviations used. ICU-Intensive Care Unit, OR-Operating Room, PACU-Post Anesthesia Care Unit, Pre-op-Pre-operative Care Unit, Pt Room-Patient's Hospital Room.

\subsection{Surgery Procedure and Anesthetic Procedure}

Obstetric/Gynecological related surgeries constituted the majority of cases (24\%), with next most common surgical specialty being orthopedic-related cases (15\%; Figure 2). As for the types of procedures, Cesarean sections had the most prevalent number of cases (Figure 4). Of the 38 cases, 26 were related to anesthesia complications and 12 were surgical complications (Figure 5).

\subsection{Cause of Injury and Outcome of Injury}

Anoxic encephalopathy (23\%) was the most common type of claimed injury followed by nerve injuries (21\%; Figure 6). Over half the cases resulted either in death (34\%) or permanent CNS damage (26\%) (Figure 7). Most injuries resulted from loss of airway (Figure 8). Over half the cases (60\%) were performed under general anesthesia with the remainder performed under regional anesthesia and unconscious sedation. Of cases involving regional anesthesia, 87\% involved neuraxial interventions.

\subsection{Care Providers Involved}

At the DMC facilities, CRNA cases accounted for approximately $68 \%(n=26)$ of incident claims with MD/ Resident anesthesiologists accounting for approximately $13 \%(n=5)$ and $18 \%(n=7)$ involved solely the MD Anesthesiologist without a resident or CRNA (Figure 9). The remaining 31 cases involved an anesthesiologist with an accompanied CRNA or resident. Of these, 5 involved a resident and the remaining involved a CRNA. Of note, of the five cases involving MD/Residents anesthesiologists, two occurred involving the same resident. Two of the anesthesiologists involved were not board certified or board eligible when the injuries took place.

In two satellite hospitals (SGH and DRH) where CRNA's cover over 85\% of OR cases in comparison to MD residents covering less than $10 \%$ of the overall case load, CRNA's were the only anesthesia providers cited in the combined four incident reports.

The suburb hospital had 19\% of reported incidents, the highest percentage in the entire DMC system. At this facility, just under $90 \%$ of the cases are covered by CRNA's and approximately $6 \%$ of cases are led by MD/ Resident anesthesiologists. Five of the six reported incidents cited CRNA's and one incident involved an MD resident anesthesiologist. Regarding recurrence, most of the cases were a one-time incident for a particular anesthesiologist. 


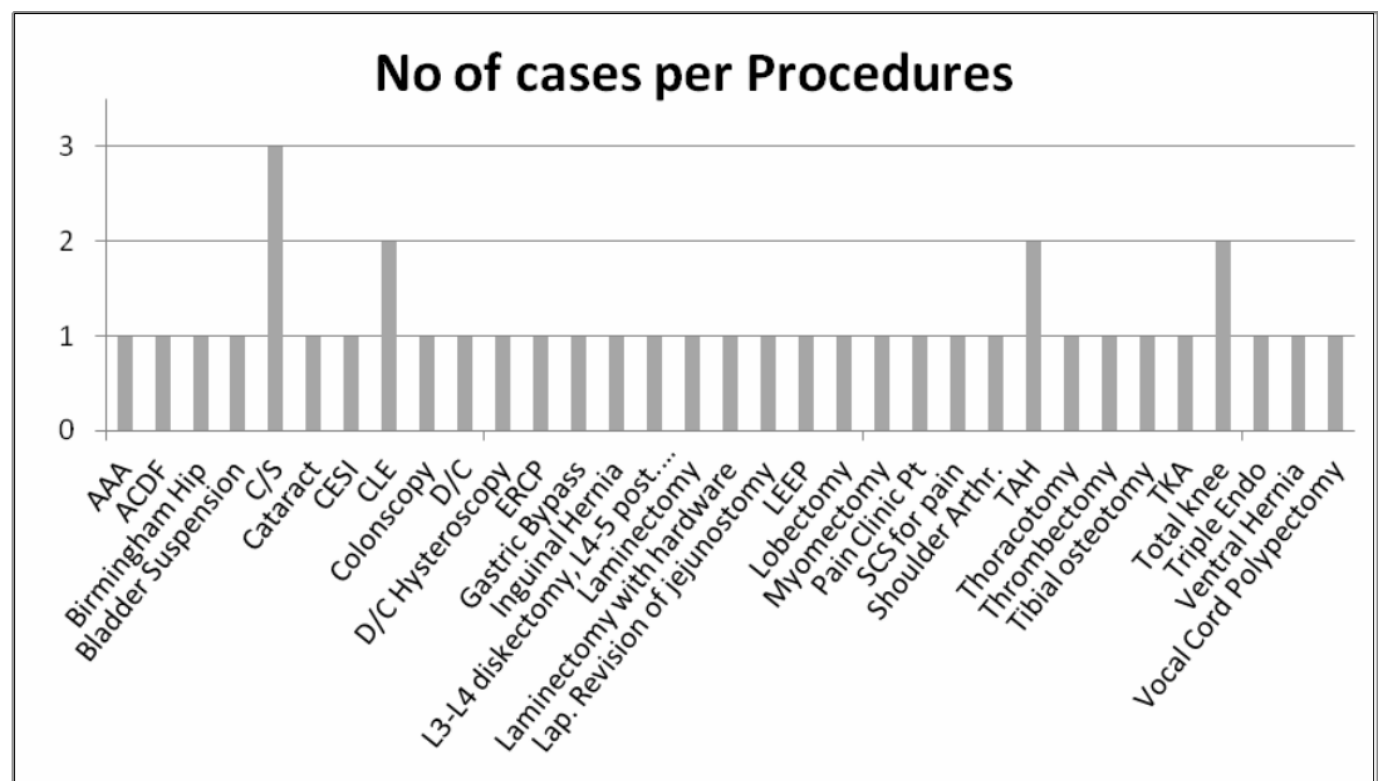

Figure 4. Litigation claims involving anesthesia care provider distribution among involved procedures at the DMC. AAA-Abdominal Aortic Aneurysm, ACDF-Anterior cervical disk fusion, C/S-Cesarean Section, CESI-Cervical epidural steroid injection, CLE-Clear Lens Extraction, D/C - Dilation and Curettage, ERCP_Endoscopic Retrograde Cholangiopancreatography, LEEP_Loop Electrosurgical Excision Procedure, SCS-Spinal Cord Stimulator, TAH-Total Abdominal Hysterectomy, TKA-Total Knee Arthroplasty, Triple Endo-Triple Endoscopy.

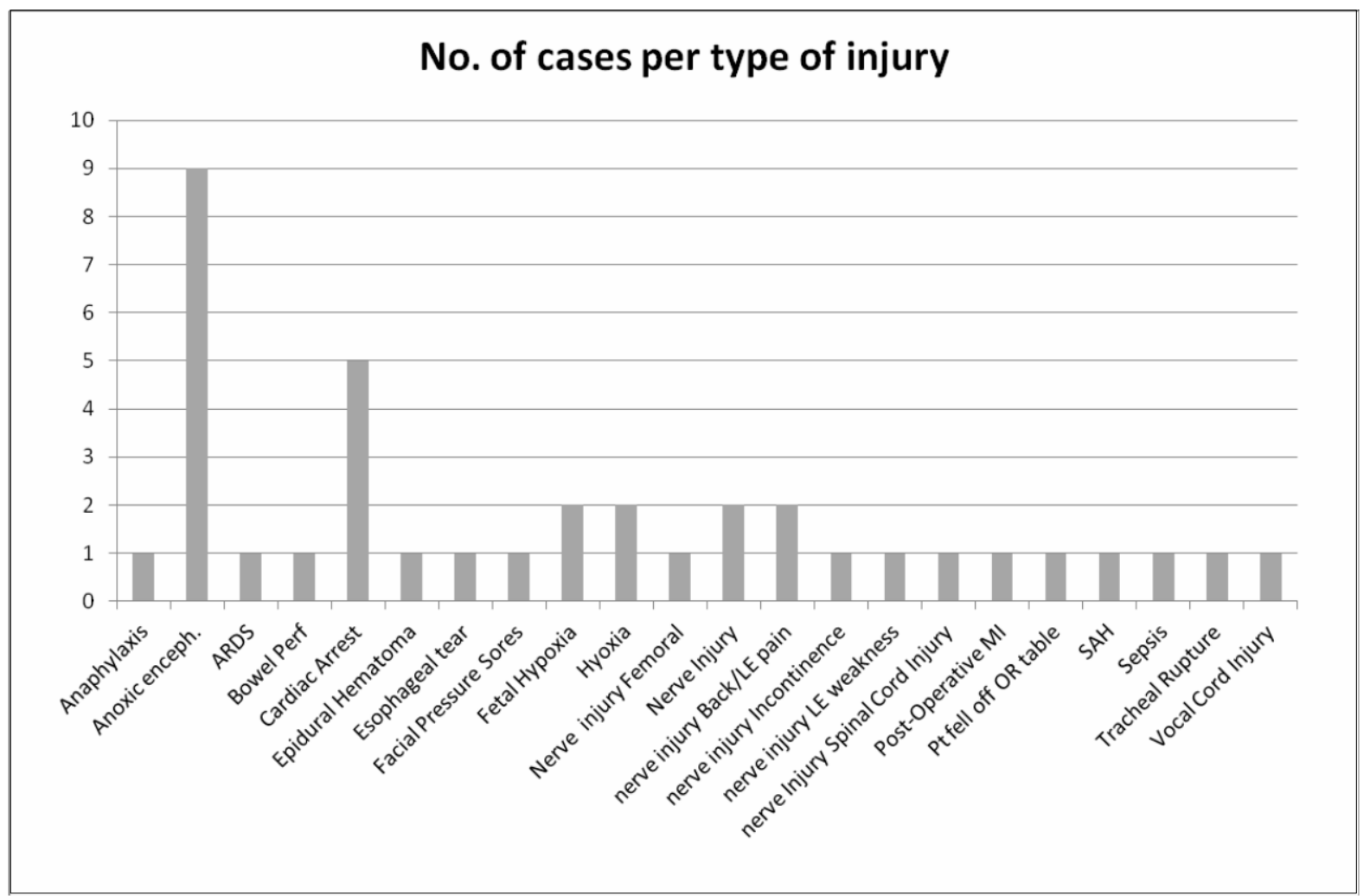

Figure 5. Litigation claims involving anesthesia care provider distribution among types of injuries at DMC. Anoxic enceph. —Anoxic Encephalopathy, ARDS-Acute Respiratory Distress Syndrome, Bowel Perf-Bowel Perforation, LE-Lower Extremity, MI-Myocardial Infarction, Pt—patient, OR-Operating Room, SAH—Subarachnoid Hemorrhage. 


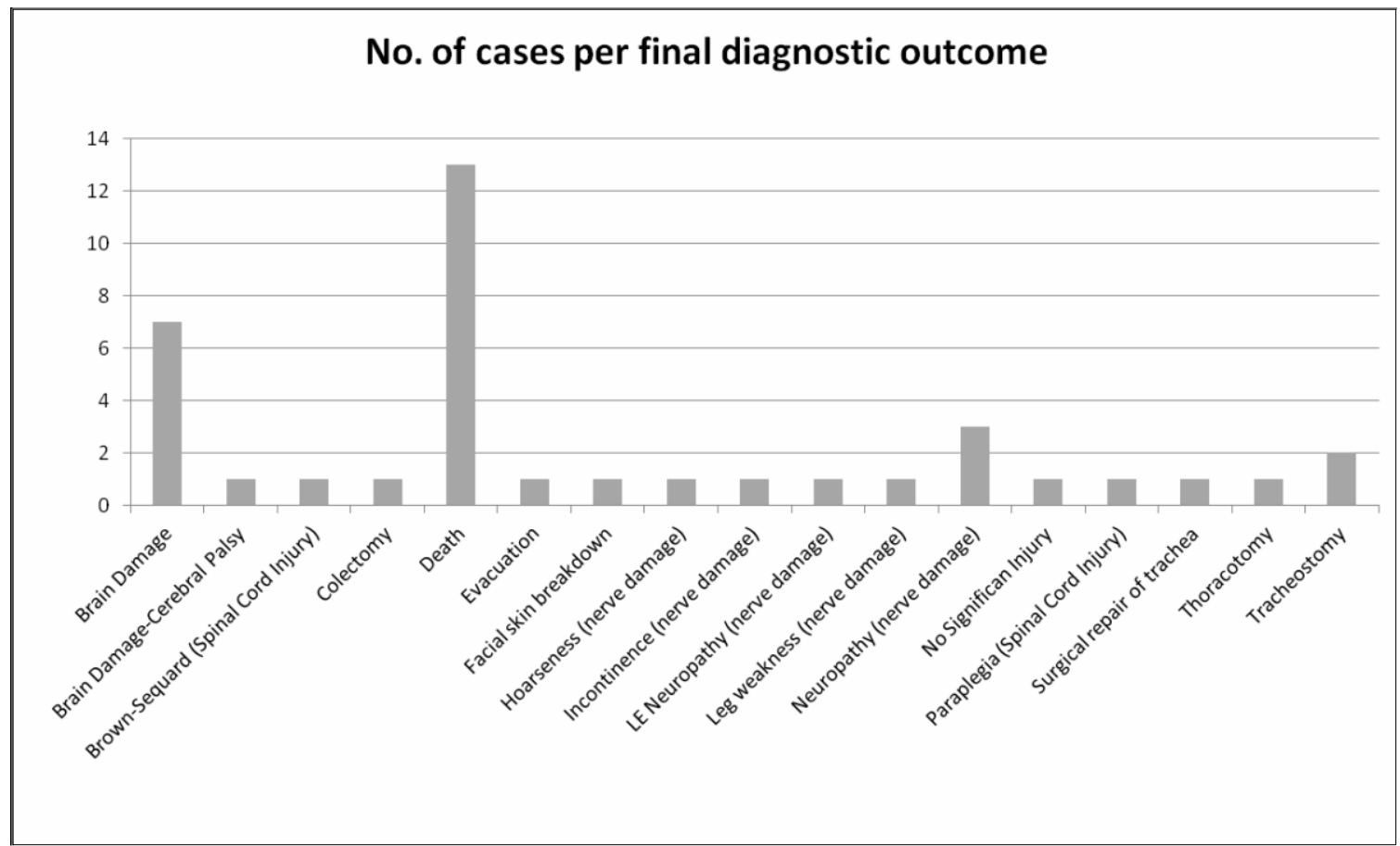

Figure 6. Litigation claims involving anesthesia care provider distribution among diagnostic outcome at the DMC.

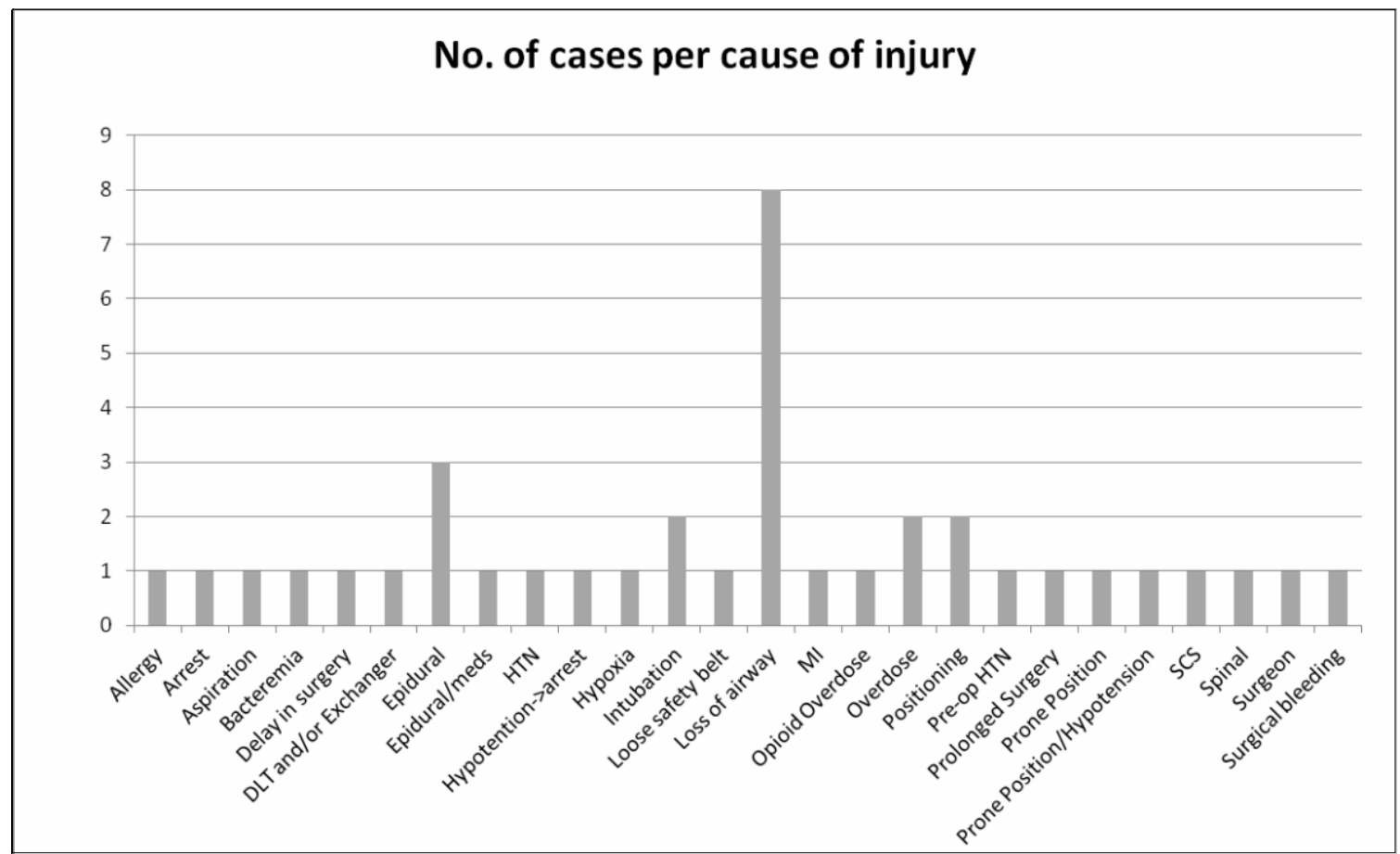

Figure 7. Litigation claims involving anesthesia care provider distribution among cause of injuries at DMC.

\subsection{Compensation/Settlements}

Of all cases file, 14 resulted in compensation to the patient or family. Eight cases are open and still pending litigation. The remaining cases were dismissed, dropped, withdrawn, or past the statute of limitation (Figure 10). One case went to the trial court and a jury panel ruled in favor of the physician. The compensations made were 


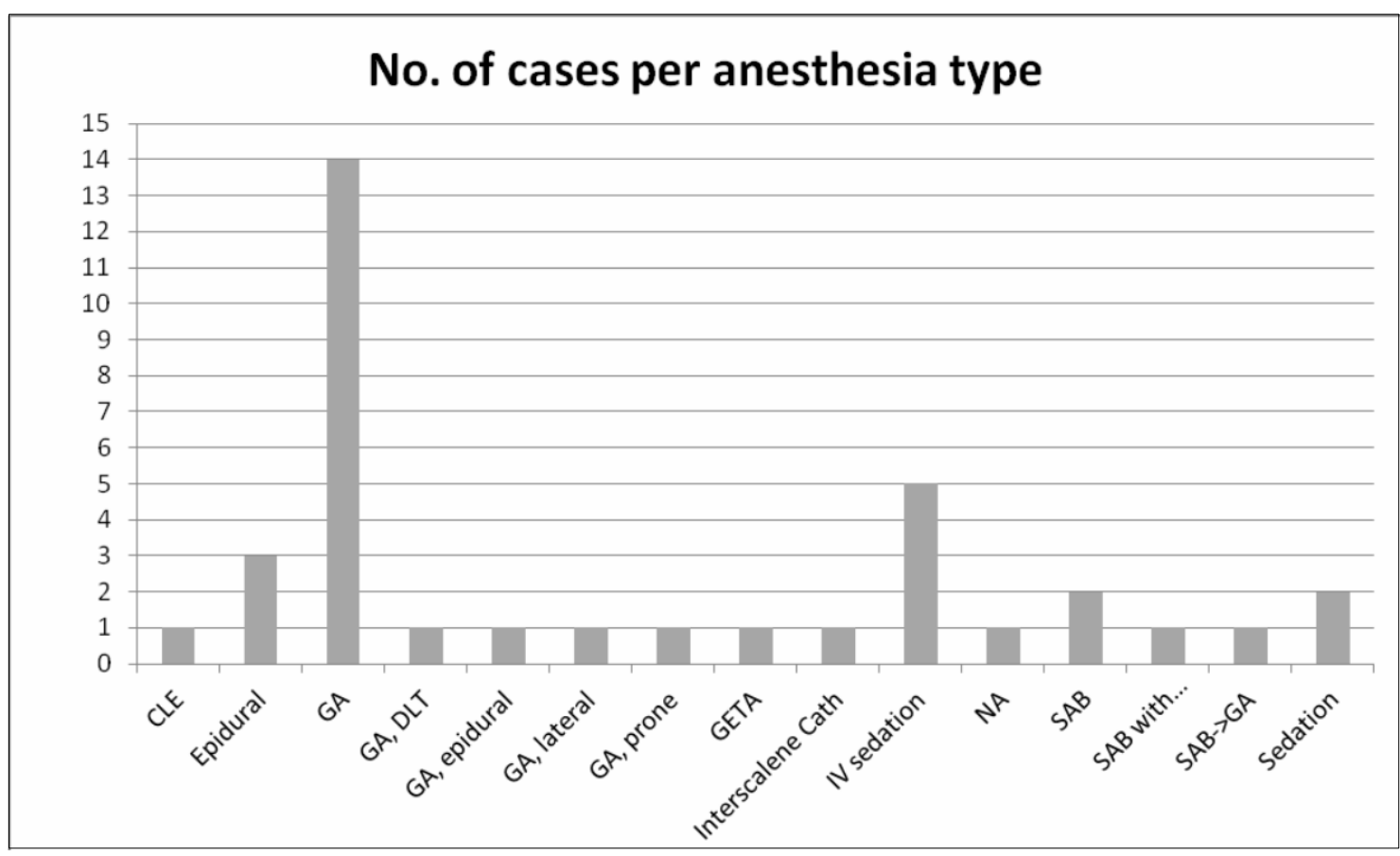

Figure 8. Litigation claims involving anesthesia care provider distribution among anesthesia type at DMC. CLE-Cesarean Labor Epidural, Epidural-Epidural Anesthesia for a non-obstetric case, GA —General Anesthesia without endotracheal tube, GA lateral-during operation, patient was lateral with endotracheal tube, GA prone-during operation, patient was prone with endotracheal tube, DLT_-Double Lumen Tube, GETA-General Endotracheal Anesthesia, IV sedation-Intra Venous sedation, SAB-Spinal Anesthesia Block, SAB with... Spinal Anesthesia Block with Regional Anesthesia, SAB -> GA Spinal Anesthesia Block which was converted to General Anesthesia, NA—local anesthesia only.

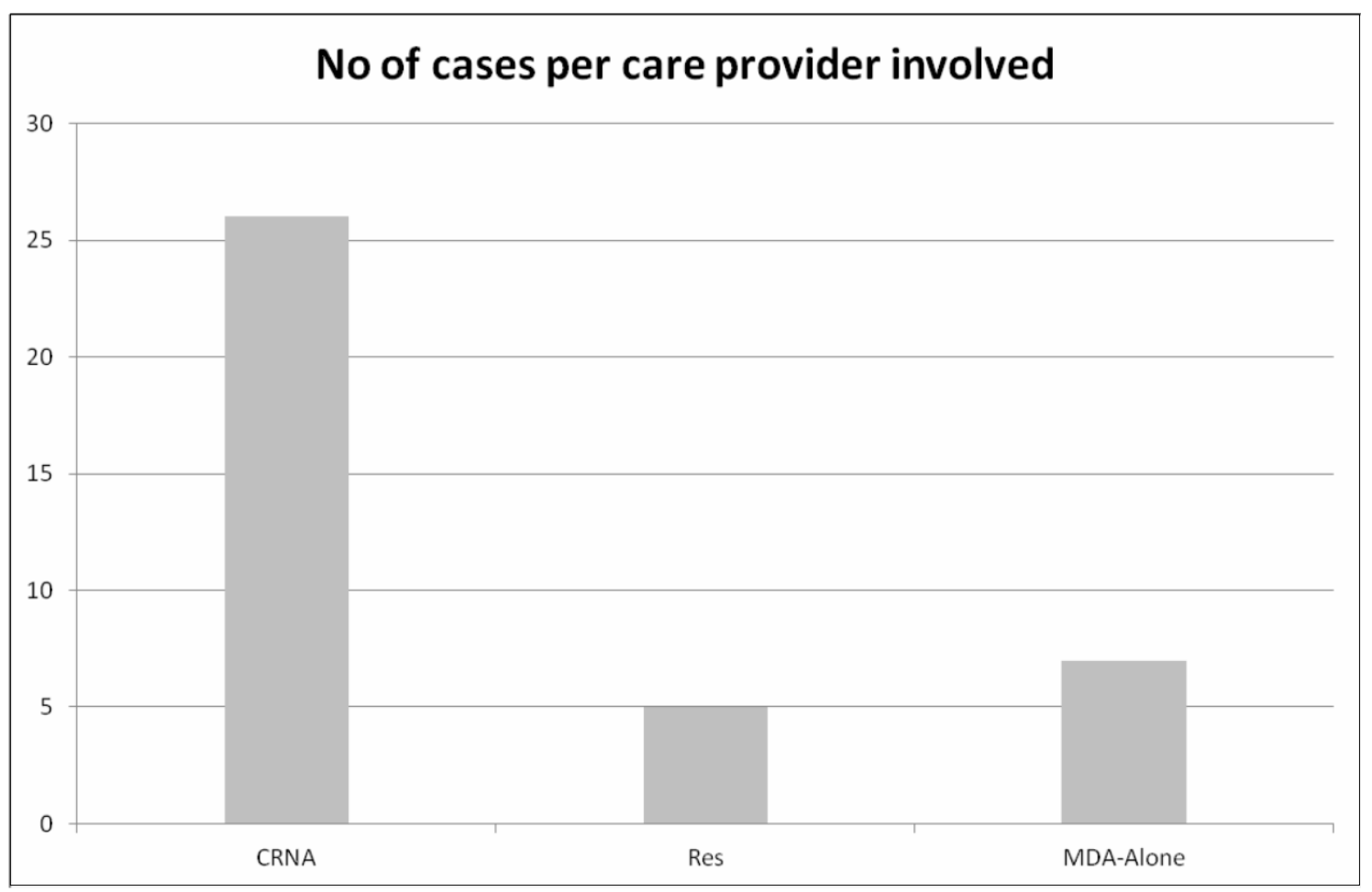

Figure 9. Litigation claims involving anesthesia care provider distribution among care provider title at DMC. 


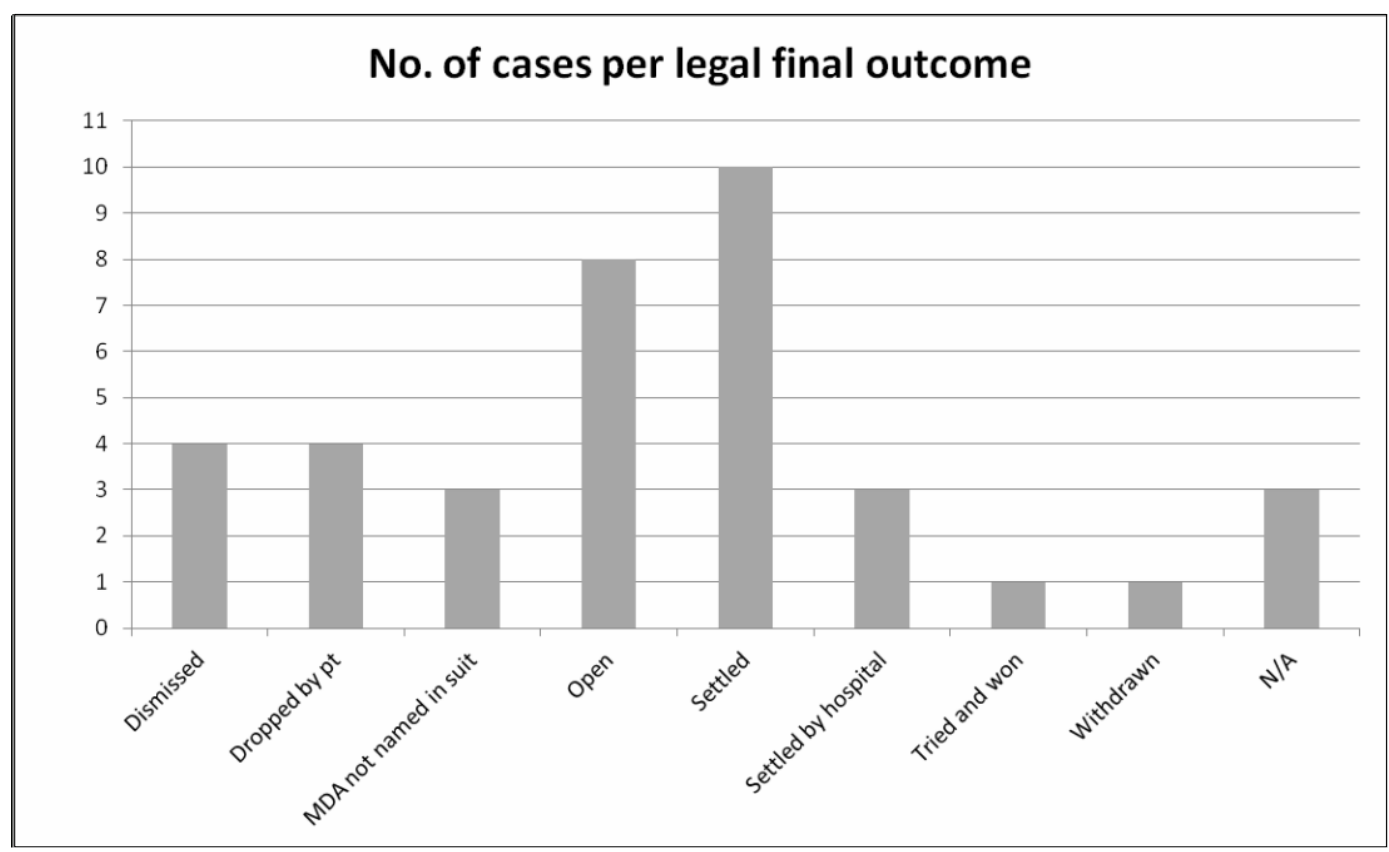

Figure 10. Litigation claims involving anesthesia care provider distribution among legal outcomes at DMC.

between $\$ 80,000$ and $\$ 400,000$, with the $25 \%$ of cases in the $\$ 200,000-\$ 250,000$ range.

\section{Discussion}

As a medical specialty, anesthesiology remains at the upper tier in terms of the cost medical malpractice claims [8]. In this study, we aimed at reporting different factors involving malpractice against anesthesiologists at the Detroit Medical Center.

As in other closed claim analyses, this study acknowledges some limitations. Closed claims data do not always reflect the occurrence frequency of outcomes or events. Some injured patients can decide not to file claims while others who were not injured do file claims [9]. The largest limitation involved gathering the detailed socio-economic data on individual plaintiffs. Since the majority of cases performed at the DMC occur at an urban hospital setting typically on patients from a lower socio-economic status. The majority of filed claims came from suburban hospitals with patient base with a middle and upper socio-economic status.

Out of a total of 67,000 cases in which an anesthesiology care provider was involved, the total number of litigation cases was 38 , which constitute $0.0567 \%$ of all cases. The majority of claims came from females and the most common age group was between 51 - 55 years. Most complaints occurred in the suburban Caucasian demographic. The time of day in which the highest risk of claims occurs was between 6 - 9 am most commonly occurring on Mondays and Tuesdays. One third of the cases occurred during the summer, with the highest incidence occurring during the month of August. Most of the cases were related to anesthesia complications. When claims were divided into anesthetic procedure, $60 \%$ of claims occurred during general anesthesia while the rest involved regional anesthesia. For injuries occurring in claims 87\% involved neuraxial intervention. Death occurred in $34 \%$ of the cases with $26 \%$ of claims resulting from CNS damage. For injury type of claims, the majority were distributed between nerves injuries and anoxic encephalopathy. When analyzing claim versus surgery category the Obstetric/Gynecology had the highest incidence followed by Orthopedic procedures. Most claim cases occurred in the OR and PACU, with the highest rate of incidence occurring in suburban hospitals. Data divided by anesthesia care provider was distributed with MD anesthesiologists responsible for 7 claims and the remaining 29 claims involving an MD anesthesia resident with or without a CRNA present. Approximately $25 \%$ of all claims resulted in compensation mostly between $\$ 200,000$ and $\$ 250,000$ whereas $50 \%$ of the cases did not result in any legal action.

In our study, the majority of people are Caucasian coming from a presumed middle class socio economic group with 11 cases involving males and the remaining 27 cases involved females. To our knowledge no studies 
have considered gender, race, ethnicity or social class. Differences in the accessibility to health care among different racial and ethnic groups often results in unequal distribution of medical malpractice cases [7]. The factors accounting for these differences are complex and include health insurance coverage, affordability due to individual income and availability of adequate medical services in cities versus suburb areas [10]. It is possible that Caucasian middle class patients are the prime litigators being more aware of the legal system with better access than patients from lower socio-economic background. Alternatively these patients may be more likely to choose to go to the more aesthetically pleasant suburban hospitals with physicians that have less exposure to complex medical issues.

We observed that the highest claim rate was filed by patients admitted to suburb hospitals affiliated with DMC. In the US, as compared to their larger counterparts, small rural hospitals are reported to provide a lower quality of health care and have their patient outcomes at the lowest scale [11]. Data from approximately 4800 hospitals serving Medicare beneficiaries were analyzed to evaluate the care quality provided by critical access hospitals (CAHs). The criteria for classifying a hospital as CAH are to have less than twenty-five acute care hospital beds and to be located thirty-five miles or more away from the closest full-service hospital (non-CAHs). In this study rural critical care hospitals had a higher 30-day patient death rates resulting from cardiac-related cases than the non-CAHs. The data was distributed as follow: $23.5 \%$ compared to $16.2 \%$ for heart attack cases, $13.4 \%$ compared to $10.9 \%$ for congestive heart failure cases and $14.1 \%$ compared to $12.1 \%$ for pneumonia cases. Notably, CAHs had a smaller chance of having adequate intensive care units [11].

Sixty percent of our study claims involved cases performed under general anesthesia while the rest involved regional anesthesia with $87 \%$ comprising neuraxial intervention. There are numerous risk factors from undergoing regional anesthesia including paresthesia following insertion of needles, hypotension, and anticoagulation [12] [13]. In a study of neurological complications following epidural anesthesia, 48 out of 32,718 cases (0.1\%) suffered from transient paralysis, with 7 patients (0.02\%) suffered from permanent paralysis. From studies compiling data for over 50,000 patients, it was reported that injuries related to spinal anesthesia ranged from between $0.08 \%-0.12 \%$ [5] [6]. In a study of the incidences of cardiac arrest and neurologic injury related to regional anesthesia occurrences were very low, but significantly increased following spinal anesthesia compared to other regional procedures [14]. In the same study two thirds of the patients with neurologic deficits had either a paresthesia during needle placement or pain on injection [14]. A retrospective study conducted by Horlocker showed similar results [13]. Our review mirrored both of these studies [13] [14].

Most of the injuries in the study claim cases resulted from loss of airway. Respiratory complications were the most frequent problem encountered with hypoxemia which was the most severe complication. Esophageal intubation, resulting in the inability to oxygenate and ventilate, accounted for over 25 percent of the claims. Since 1985, ASA-CCP has been collecting data and has already collected 30 years data of anesthesia malpractice claims. Nine hundred claims revealed 14 cardiac arrest deaths during spinal anesthesia presumably due to undetected respiratory insufficiency and sympathetic blockade [15]. Sympathetic system blockage by spinal anesthetics followed by a vasovagal response induces an imbalance between the parasympathetic/sympathetic systems which is likely exacerbated in susceptible individuals with increased vagal tone which is estimated to be in $7 \%$ of the population [16].

We observed that Obstetric/Gynecological procedures had the highest rate of the litigation cases. Maternal deaths account for $19 \%$ to $22 \%$ of all obstetric claims. The most common event related to maternal death was failure to secure an airway. Forty seven percent of all obstetric claims outcomes were head and back pain beside emotional anxiety [17].

Regarding anesthesia provider titles, one of the most expensive areas involves supervision issues, mainly supervision of non-physicians for whom the anesthesiologist is held liable. Anesthesiologists are often not primarily involved in delivering anesthesia care. The majority of these cases involve a CRNA and anesthesiology assistant (AA). Malpractice cases could be due to both clinical and administrative factors such as overload of responsibility, diffusion of responsibility, lack or inadequate physician supervision, absence or deviation of written protocols by physician extenders, and failure or delay in seeking physician collaboration by physician extenders. According to the ASA, anesthesiologists are more frequently pressured to alter their practices, by hospitals which are held, by court ruling, responsible for their practices [18].

The professional liability closed claim data collected by the Physician Insurers Association of America (PIAA) observed that the number of medical malpractice claims reported have not significantly increased over the past 20 years, the average indemnity payment for anesthesiology claims has significantly jumped from $\$ 93,537$ in 
1985 to $\$ 403,095$ in 2005, this is more than double even accounting for inflation. On average 33 percent of closed claims were paid, with an indemnity average of $\$ 362,880$. Within these twenty years, claims with a higher patient injury severity have also skyrocketed. These claims refer to a severity of death, grave, or major permanent injury where the last two account for the highest compensation because they require extended and expensive specialized care [19].

Numerous malpractice claims attributed to non-physicians refer mostly to inadequate physician supervision, lack of communication between the anesthesia care providers and absence of clear protocols which makes the presence of supervision protocols essential during intra-operative monitoring. Upon analysis of our study, institutional protocols have been implemented to minimize physician extenders on working overnight. This overnight staffing is now covered by resident anesthesiologists. Furthermore, it has been recommended and implemented that higher acuity cases be assigned to MD anesthesiologists teamed with resident anesthesiologist in order to minimize the early shift time and early week complications. Additionally, it was recommended that more patient safety-related information is communicated between surgeons, anesthesiologists and physician extenders so that potential patient safety issues can be identified more rapidly with a group approach to facilitating more optimal patient outcomes.

\section{Funding}

This project was supported by intra-departmental funding. No other sources of funding were used for this project.

\section{References}

[1] Kesselheim, A.S. (2010) Permitting Product Liability Litigation for FDA-Approved Drugs and Devices Promotes Patient Safety. Clinical Pharmacology \& Therapeutics, 87, 645-647. http://dx.doi.org/10.1038/clpt.2009.213

[2] Karch, D.L., Logan, J. and Patel, N. (2011) Surveillance for Violent Deaths-National Violent Death Reporting System, 16 States, 2009. Morbidity and Mortality Weekly Report (MMWR) Surveillance Summaries, 60, 1-49.

[3] Caplan, R.A., Posner, K.L., Ward, R.J. and Cheney, F.W. (1990) Adverse Respiratory Events in Anesthesia: A Closed Claims Analysis. Anesthesiology, 72, 828-833. http://dx.doi.org/10.1097/00000542-199005000-00010

[4] Peterson, G.N., Domino, K.B., Caplan, R.A., Posner, K.L., Lee, L.A. and Cheney, F.W. (2005) Management of the Difficult Airway: A Closed Claims Analysis. Anesthesiology, 103, 33-39. http://dx.doi.org/10.1097/00000542-200507000-00009

[5] Hove, L.D., Nielsen, H.B. and Christoffersen, J.K. (2006) Patient Injuries in Response to Anaesthetic Procedures: Cases Evaluated by the Danish Patient Insurance Association. Acta Anaesthesiologica Scandinavica, 50, 530-535. http://dx.doi.org/10.1111/j.1399-6576.2006.001024.x

[6] Currie, M., Webb, R.K., Williamson, J.A., Russell, W.J. and Mackay, P. (1993) The Australian Incident Monitoring Study. Clinical Anaphylaxis: An Analysis of 2000 Incident Reports. Anaesthesia and Intensive Care, 21, 621-625.

[7] Nelson, A.R. (2003) Unequal Treatment: Report of the Institute of Medicine on Racial and Ethnic Disparities in Healthcare. The Annals of Thoracic Surgery, 76, S1377-S1381. http://dx.doi.org/10.1016/S0003-4975(03)01205-0

[8] Domino, K.B., Posner, K.L., Caplan, R.A. and Cheney, F.W. (1999) Airway Injury during Anesthesia: A Closed Claims Analysis. Anesthesiology, 91, 1703-1711. http://dx.doi.org/10.1097/00000542-199912000-00023

[9] Cheney, F.W. (1999) The American Society of Anesthesiologists Closed Claims Project: What Have We Learned, How Has It Affected Practice, and How Will It Affect Practice in The Future? Anesthesiology, 91, 552-556. http://dx.doi.org/10.1097/00000542-199908000-00030

[10] Mayberry, R.M., Mili, F. and Ofili, E. (2000) Racial and Ethnic Differences in Access to Medical Care. Medical Care Research and Review, 57, 108-145. http://dx.doi.org/10.1177/1077558700574006

[11] Joynt, K.E., Harris, Y., Orav, E.J. and Jha, A.K. (2011) Quality of Care and Patient Outcomes in Critical Access Rural Hospitals. The Journal of American Medical Association, 306, 45-52.

[12] Scott, D.B. and Hibbard, B.M. (1990) Serious Non-Fatal Complications Associated with Extradural Block in Obstetric Practice. British Journal of Anaesthesia, 64, 537-541. http://dx.doi.org/10.1093/bja/64.5.537

[13] Horlocker, T.T., McGregor, D.G., Matsushige, D.K., Schroeder, D.R. and Besse, J.A. (1997) A Retrospective Review of 4767 Consecutive Spinal Anesthetics: Central Nervous System Complications. Perioperative Outcomes Group. Anesthesia \& Analgesia, 84, 578-584.

[14] Auroy, Y., Narchi, P., Messiah, A., Litt, L., Rouvier, B. and Samii, K. (1997) Serious Complications Related to Re- 
gional Anesthesia: Results of a Prospective Survey in France. Anesthesiology, 87, 479-486. http://dx.doi.org/10.1097/00000542-199709000-00005

[15] Caplan, R.A., Ward, R.J., Posner, K. and Cheney, F.W. (1998) Unexpected Cardiac Arrest during Spinal Anesthesia: A Closed Claims Analysis of Predisposing Factors. Anesthesiology, 68, 5-11. http://dx.doi.org/10.1097/00000542-198801000-00003

[16] Thrush, D.N. and Downs, J.B. (1999) Vagotonia and Cardiac Arrest during Spinal Anesthesia. Anesthesiology, 91, 1171-1173. http://dx.doi.org/10.1097/00000542-199910000-00037

[17] Crawforth, K. (2002) The AANA Foundation Closed Malpractice Claims Study: Obstetric Anesthesia. AANA Journal, 70, 97-104.

[18] Reducing Medical Malpractice Liability: Anesthesiology Preventive Action and Loss Reduction Plan. First Professional Insurance Company/APAC, Risk Management Department.

[19] Weiler, P.C. (2005) A Measure of Malpractice: Medical Injury, Malpractice Litigation, and Patient Compensation. Harvard University Press, Cambridge. 